Research Article

\title{
Optimal Operation Strategy of Flexible Interconnected Distribution Network Based on SES-VSC-MTDC
}

\author{
Le Ge ${ }^{D},{ }^{1}$ Limin $\mathrm{Lu}^{2}{ }^{2}$ Xiaodong Yuan, ${ }^{3}$ and Yongzhou $\mathrm{Yu}^{1}$ \\ ${ }^{1}$ School of Electrical Engineering, Nanjing Institute of Technology, Nanjing 211167, China \\ ${ }^{2}$ Changzhou Power Supply Company, State Grid Jiangsu Electric Power Co., Ltd., Changzhou 213000, China \\ ${ }^{3}$ Electric Power Research Institute, State Grid Jiangsu Electric Power Co., Ltd., Nanjing 211103, China
}

Correspondence should be addressed to Le Ge; supertiger_bear@126.com

Received 17 April 2020; Accepted 20 July 2020; Published 24 August 2020

Academic Editor: Luis J. Yebra

Copyright ( $92020 \mathrm{Le}$ Ge et al. This is an open access article distributed under the Creative Commons Attribution License, which permits unrestricted use, distribution, and reproduction in any medium, provided the original work is properly cited.

\begin{abstract}
The increasing integration of renewable energy is challenging the secure operation of the power system. System flexibility or the capability to address the significant power fluctuations from renewable energy is becoming more and more relevant. Self-energy storage-based multiterminal back-to-back VSC-HVDC (SES-VSC-MTDC) technology is first proposed, and it can realize the power regulation on both temporal and spatial dimensions, which helps improve the power supply reliability and the capacity to accommodate renewable energy of the interconnected distribution networks. Then, to address the coordination control problem of the energy storage and back-to-back VSC-HVDC, a comprehensive control strategy of SES-VSC-MTDC is proposed based on the optimal power flow preprocessing and state of charge interval division. Then, the power regulation model and the energypower regulation timing model of SES-VSC-MTDC are established for different control strategies. Then, we use the primal-dual interior-point method to solve the developed optimal operation model of flexible interconnected distribution network. Finally, a 33-bus system with four interconnected feeders is used to test the effectiveness of the SES-VSC-MTDC technology and its operation control strategy.
\end{abstract}

\section{Introduction}

High reliable power supply and full accommodation for large-scale renewable energy mean high requirement for the construction and operation of distribution network $[1,2]$. The loop operation of distribution network is an effective method to improve the power supply reliability. However, the large impulse and the closed-loop current caused by the AC interconnection directly impact the stable operation of distribution networks [3]. The voltage violations caused by the power fluctuations of renewable energy are increasingly significant. The increasing distribution network capacity and the rising integration of reactive power compensation devices such as static VAR generator (SVG) [4] will significantly increase the construction and operation cost. Although the active power limit and the reactive power regulation of the inverter can properly solve the voltage violations [5], the consequent abandonment of wind and solar energy in the distribution network decreases the utilization level of renewable energy.

Multiterminal back-to-back VSC-HVDC (VSC-MTDC) is a novel power flow flexible control technology to solve the above problems. Its operation characteristics are similar to the soft open point (SOP) [6-9], the DC links [10], and the flexible interconnected controller [11]. The AC systems are decoupled and interconnected (AC-DC-AC) based on the voltage source converters (VSCs) with common DC bus, which can realize long-term secure loop operation of any different feeders. Four-quadrant PQ control can accurately regulate the power flow of the interconnected distribution network. To reduce the cost and complexity of the control system, the omission of DC lines is more applicable to the actual distribution network [3, 6-9]. Li et al. [8] proposed a combined decentralized and local voltage control strategy of SOPs to rapidly cope with the frequent voltage fluctuations. Based on the dynamic network partition results, the 
decentralized optimization is applied to regulate the active power transmission of SOPs among the connected areas. Shafik et al. [9] proved that technical, economic, and environmental perspectives can be achieved together for a real case study from Egypt through optimal system operation utilizing SOP devices and distributed renewable sources of energy.

In the power level, the flexible power flow control technology is essential to the power regulation. In the energy level, the power grid provides the "energy container" on the spatial dimension. However, when the available capacity of interconnected feeder is low, the impact of optimal operation on the interconnected distribution network will be reduced, and even the system security and power quality constraints cannot be ensured.

Energy storage system (ESS), as the "energy container" on the temporal dimension, essentially improves the simultaneous problems of power generation, transmission, and consumption. Restricted by the development of material science and technology, ESS is still relatively expensive and cannot replace the power flow control equipment completely in the near future. The optimizing complementary problem between ESS and VSC-MTDC is worthy of further study [12]. The "AC-DC-AC" operation mode of back-to-back VSC-HVDC provided the possibility of integration of ESS and VSC-MTDC [13]. SOP integrated with ESS is simulated, and the prototype was preliminarily tested [14]. Ji et al. [15] compared and analyzed maximum hosting capacity of DG based on the conventional SOP and the SOP integrated with ESS in the distribution network. Yao et al. [16] verified that the SOP integrated with ESS can significantly reduce network losses. However, the above research studies did not discuss the coordination control of the energy storage and SOP in depth, and the cost of energy storage in the system was not considered.

From the perspective of reducing configuration capacity of ESS and improving its operation life, this paper studies the SES-VSC-MTDC comprehensive control strategy based on the optimal power flow preprocessing and ESS SOC (state of charge) interval division, models and analyzes its power regulation and power-energy timing regulation, and proposes an optimal operation strategy of flexible interconnected distribution network. Through the analysis of simulation results, it is shown that SES-VSC-MTDC can dynamically adjust power flows according to the change of distribution network operation states and quickly respond to fluctuations of DGs and loads, which can make full use of renewable energy, improve power supply reliability, improve power supply quality, and reduce comprehensive power supply cost.

\section{SES-VSC-MTDC Composite Control Strategy}

2.1. The Working Principle of SES-VSC-MTDC. The structure of the flexible interconnected distribution network based on SES-VSC-MTDC is shown in Figure 1. The DC sides of multiterminal AC/DC converters and DC/DC converters of ESS are connected in parallel to the common DC bus. The AC sides are, respectively, connected to each feeder of the distribution network to realize flexible interconnection (i.e., AC-DC-AC decoupling) of distribution network. The DC/ DC converters implement charge and discharge control of ESS, which enables the SES-VSC-MTDC to increase the energy timing regulation capability and become a highly integrated comprehensive energy transformation device. Under normal operating conditions, each port of SES-VSCMTDC realizes flexible active power exchange between different feeders and implements independent control of the reactive power according to the optimal operation dispatching instruction. When the fault occurs in a feeder or $\mathrm{HV}$ power grid, the rapid switching of control modes of multiterminal converters can be realized, and the real-time transfer of nonfaulty area load can be ensured and power supply reliability can be improved.

Compared with the VSC-MTDC, the SES-VSC-MTDC proposed in this paper is applicable for the scenario with high-penetration renewable energy. Considering the cost, the configuration capacity and charge-discharge cycle number of the ESS should be minimized. Hence, only when the available capacity of interconnected feeders is insufficient to meet system power supply reliability and the full accommodation of renewable energy, the ESS is put into operation seamlessly according to the control strategy.

2.2. SES-VSC-MTDC Comprehensive Control Strategy. The comprehensive control strategy with the SES-VSCMTDC is based on the power regulation, and ESS is put into operation only when VSC-MTDC cannot meet the power system security and power quality constraints. The SOC of the ESS has the strict continuity in time series. To avoid overcharge or overdischarge and ensure that the ESS can be adjusted in the next periods, it is necessary to properly divide the operation states of ESS and control the charging or discharging processes of the ESS.

First, the interval of SOC is divided into the standby state, the normal and nonstandby state, and the abnormal state.

(i) Standby state: SOC is moderate, and the ESS can meet the optimal operation requirements of distribution network in the next period, and the SOC interval is set to be $[0.4,0.6]$.

(ii) Normal and nonstandby state: the available capacity of ESS is relatively small due to the security margin, and the SOC interval is set to be $[0.15,0.4]$ and $[0.6$, 0.85].

(iii) Abnormal state: the SOC recovery should be performed immediately, and the SOC interval is set to be $[0,0.15]$ and $[0.85,1]$.

The comprehensive control strategy includes the power regulation optimization (control strategy I), the energypower timing regulation optimization (control strategy II), and the ESS SOC recovery (control strategy III).

(i) Control strategy I: the ESS is out of operation, and the SES-VSC-MTDC only provides the power regulation. 


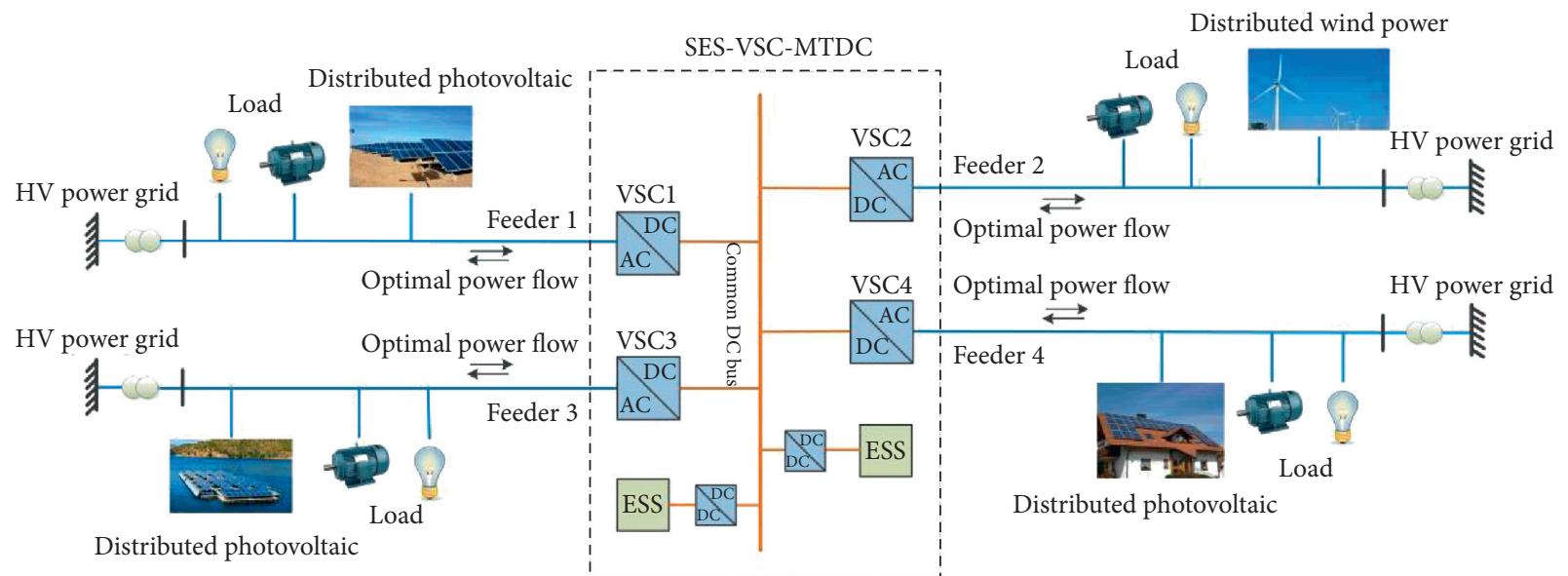

FIGURE 1: Schematic diagram of flexible interconnected distribution network based on SES-VSC-MTDC.

(ii) Control strategy II: the ESS is in operation, and the SES-VSC-MTDC provides the cooperative regulation of power and energy.

(iii) Control strategy III: the ESS is in operation, the SOC of the ESS is recovered, and the VSC-MTDC independently provides power regulation.

Based on above analyses, it is first pretreated to determine whether there is a solution to the optimization calculation of the interconnected distribution network without energy storage. Then, as shown in Table 1, the corresponding control strategies are applied according to the various SOC intervals. The overall control process is shown in Figure 2. The specific technical methods of pretreatments are described in Sections 3.1 and 4.1. The complete optimization strategy of the flexible interconnected distribution network based on the SES-VSC-MTDC is given in Sections 3 and 4.

It is noteworthy that the above analysis is only for the steady-state operation. When one or more converters fail or the grid fails seriously, it is necessary to study the coordinated control of multiple converters and the seamless switching of their control modes. Relevant research can be found in [17].

\section{SES-VSC-MTDC Operation Models}

3.1. Power Regulation Model. Under normal working conditions of the distribution network, the master converter of SES-VSC-MTDC adopts the $U_{\mathrm{dc}} Q$ control, and other slave converters adopt the PQ control [18]. The master converter balances the active power of the grid to maintain the DC bus voltage. In order to realize the decoupling control of the active and reactive power, the synchronous $d-q$ reference frame is adopted and the $d$-axis of the reference frame is aligned with the grid voltage vector. Therefore, $U_{\mathrm{sq}}=0$ and $U_{\text {sd }}=U_{s}$; the active and reactive power of the converter can be expressed as
TABLE 1: Classification of control strategies for SES-VSC-MTDC.

\begin{tabular}{lccc}
\hline No. & Pretreatments & Interval of SOC & Control strategy \\
\hline 1 & Has a solution & Standby & I \\
2 & Has a solution & Normal and nonstandby & III \\
3 & Has a solution & Abnormal & III \\
4 & No solution & Standby & II \\
5 & No solution & Normal and nonstandby & II \\
6 & No solution & Abnormal & III \\
\hline
\end{tabular}

$$
\begin{gathered}
P=\frac{3}{2} U_{\mathrm{sd}} i_{d} . \\
Q=-\frac{3}{2} U_{\mathrm{sd}} i_{q},
\end{gathered}
$$

where $i_{d}$ and $i_{q}$ are, respectively, the $d$-axis and $q$-axis components of the grid side current of the converter and $U_{\text {sd }}$ is the $d$-axis component of the AC-side voltage of the converter.

According to (1) and (2), the active and reactive power can be independently controlled by changing the converter current of the $d-q$ axis component. The converter has the equal active power at both AC and DC ends, and we can obtain

$$
\mathrm{CU}_{\mathrm{dc}} \frac{\mathrm{d} U_{\mathrm{dc}}}{\mathrm{d} t}=\sum_{i=1}^{\mathrm{N}_{\mathrm{VSC}}} \frac{3}{2} U_{\mathrm{sd} i} i_{\mathrm{d} i}+P_{\text {loss }},
$$

where $\mathrm{N}_{\mathrm{VSC}}$ is the total number of converters for the SESVSC-MTDC; $C$ is DC-side capacitor; $U_{\mathrm{dc}}$ is DC bus voltage; and $P_{\text {loss }}$ is loss of converters.

Due to the isolation of the DC link, the reactive power output of the converter does not affect each other, so that only the capacity constraints of converters need to be met. Therefore, the mathematical model is shown as follows: 


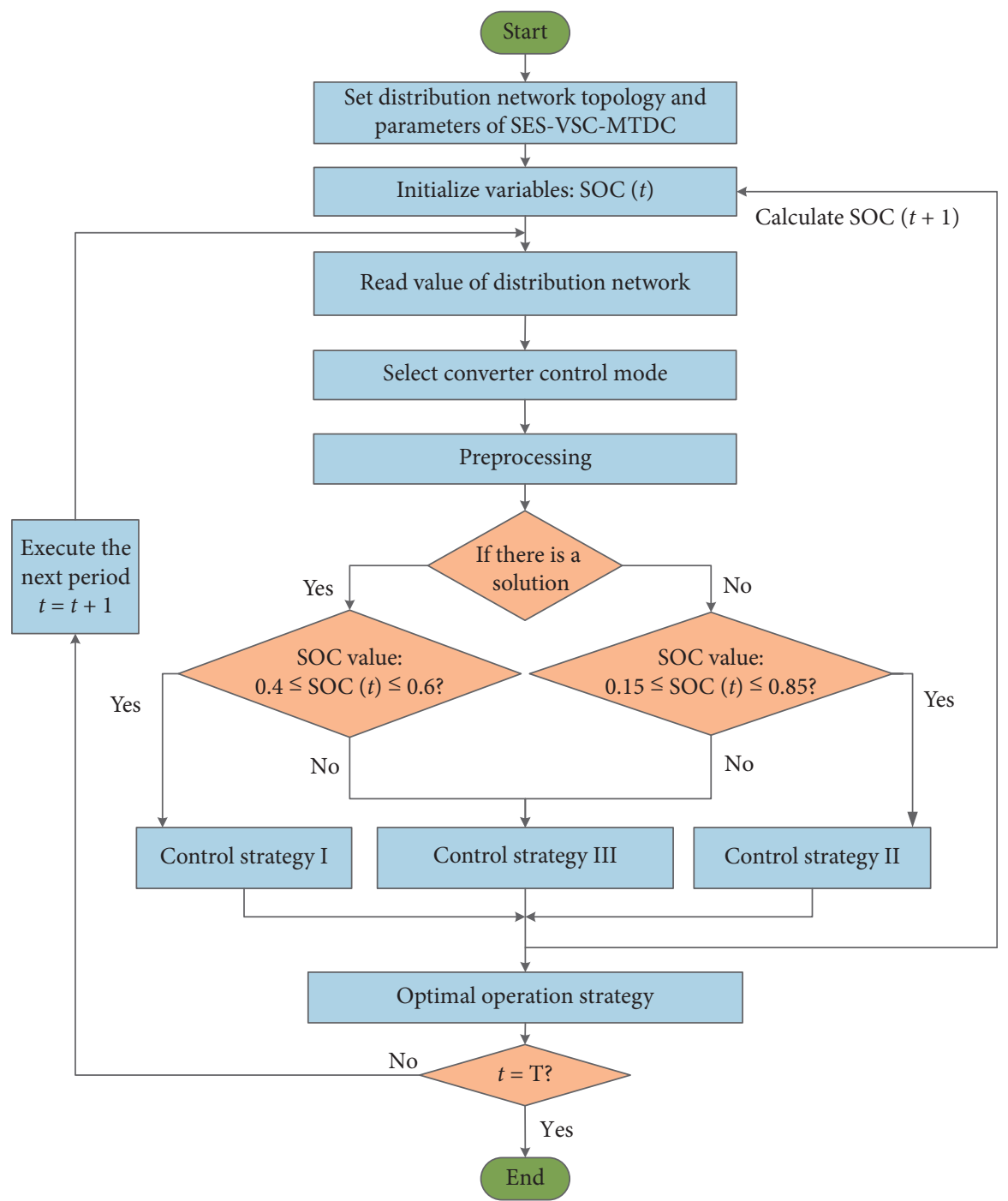

FIGURE 2: Flowchart of SES-VSC-MTDC comprehensive control.

$$
\left\{\begin{array}{l}
\sum_{k=1}^{\mathrm{N}_{\mathrm{VSC}}} P_{k}(t)+\sum_{k=1}^{\mathrm{N}_{\mathrm{VSC}}}\left(A_{k} P_{k}(t)\right)=0, \\
\left|P_{k}(t)\right| \leq \bar{P}_{k}, \\
\sqrt{P_{k}(t)^{2}+Q_{k}(t)^{2}} \leq S_{k}, \\
U_{\mathrm{dc}}=U_{\mathrm{dc}, \mathrm{ref}}
\end{array}\right.
$$

where $P_{k}(t)$ and $Q_{k}(t)$ are, respectively, the active and reactive power of the $k$-th converter at time $t$; the power injecting into the DC bus is defined as positive; $A_{k}$ is the loss factor of the $k$ th converter; $\bar{P}_{k}$ is the active power limit of the $k$-th converter; $S_{k}$ is the rated capacity of the $k$-th converter; $U_{\mathrm{dc}}$ is DC bus voltage; and $U_{\mathrm{dc}, \text { ref }}$ is the reference voltage of DC bus.

When there is power grid failure, the control requirements of the master converter are unchanged, and the faultside converter is equivalent to supplying power to the passive network, whose control object is to maintain the AC voltage amplitude and frequency. There are two types of situations:

(i) When the feeder or the HV power grid connected to the slave converter fails, the slave converter on the faulty end switches to the $U_{\text {ac }} f$ control, and the other slave converters still adopt the PQ control.

(ii) When the feeder or the HV power grid connected to the master converter fails, any slave converter can switch to the $U_{\mathrm{dc}} \mathrm{Q}$ control and become a new master converter, and the original master converter becomes the slave converter and switches to the $U_{\mathrm{ac}} f$ control. Other slave converters still adopt the PQ control.

Then, the mathematical model is further given by

$$
\left\{\begin{array}{l}
U_{\mathrm{ac}}=U_{\mathrm{ac}, \mathrm{ref}}, \\
f=50 \mathrm{~Hz},
\end{array}\right.
$$

where $U_{\mathrm{ac}}$ is the AC voltage of the fault-side converter and $U_{\mathrm{ac}, \mathrm{ref}}$ is the rated voltage of the grid (i.e., reference voltage).

3.2. Power-Energy Timing Regulation Model. Under the normal working condition of the distribution network, the master converter of the SES-VSC-MTDC still adopts the $U_{\mathrm{dc}} Q$ control, and ES converter adopts the PQ control as the 
slave converter. Therefore, the power balance equation in (4) is revised by the following equation:

$$
\sum_{k=1}^{\mathrm{N}_{\mathrm{VSC}}} P_{k}(t)+\sum_{k=1}^{\mathrm{N}_{\mathrm{VSC}}}\left(A_{k} P_{k}(t)\right)+P_{\mathrm{ESS}}(t)=0 .
$$

The control variables of the power-energy timing regulation model increase the ESS output on the basis of the power regulation model, and the corresponding chargedischarge power constraints as given by (7) and (8) and the
SOC constraints as given by (9) and (10) are added to the constraints. The output range of the active power of the ESS $P_{\text {ESS }}(t)$ will be added to the optimal operation model of the distribution network or to the ESS SOC recovery model as constraints. Then, the SOC value of ESS will be calculated again to provide constraints for the optimal decision for the next period. The ESS charge-discharge power constraints are given below:

$$
\begin{gathered}
P_{\mathrm{ESS}}(t)=u P_{\mathrm{cd}}(t), \quad u \in\{-1,0,1\} . \\
\left\{\begin{array}{l}
0 \leq P_{\mathrm{cd}}(t) \leq \min \left\{P_{\mathrm{dis}, \max }, \frac{\eta_{\mathrm{dis}} S_{\mathrm{ESS}}\left[\mathrm{SOC}(t)-\mathrm{SOC}_{\mathrm{min}}\right]}{\Delta t}\right\}, \quad u=1, \\
0 \leq P_{\mathrm{cd}}(t) \leq \min \left\{P_{\mathrm{ch}, \max }, \frac{S_{\mathrm{ESS}}\left[\mathrm{SOC}_{\mathrm{max}}-\operatorname{SOC}(t)\right]}{\eta_{\mathrm{ch}} \Delta t}\right\}, \quad u=-1 .
\end{array}\right.
\end{gathered}
$$

The ESS SOC constraints are given below:

$$
\begin{gathered}
\operatorname{SOC}(t+\Delta t)=\operatorname{SOC}(t)-\frac{P_{\mathrm{ESS}} \Delta t}{S_{\mathrm{ESS}}} \\
\operatorname{SOC}(0)=\operatorname{SOC}(T),
\end{gathered}
$$

where $\Delta t$ is the time interval; $T$ is the number of time intervals divided by a complete scheduling cycle; $P_{\mathrm{ESS}}(t)$ is the output power of the ESS at time instant $t ; P_{\mathrm{cd}}(t)$ is the charge or discharge power of ESS, which is always positive; $u$ is the state of the ESS, whose values are $-1,0$ and 1 representing three states of charge, standby and discharge, respectively; $P_{\mathrm{ch} \text {, max }}$ and $P_{\mathrm{dis} \text { max }}$ are the maximum charging and discharging power of the ESS; $S_{\mathrm{ESS}}$ is the rated power of the ESS; SOC $(t)$ is the state of charge of ESS; $S_{\text {max }}$ and $S_{\text {min }}$ are the upper and lower limits of the SOC value of the ESS, respectively; and $\eta_{\mathrm{ch}}$ and $\eta_{\text {dis }}$ are the charging and discharging efficiency of the ESS, respectively.

Under fault conditions of the distribution network, the regulation process is similar to the power regulation model as discussed above.

In the SES-VSC-MTDC system, there are three control modes of converter: the $U_{\mathrm{dc}} Q$ control, the PQ control, and the $U_{\text {ac }} f$ control. The three control methods adopt the double closed-loop control structure, and the current inner loop adopts the current control based on feed-forward decoupling. However, the outer loop of the $U_{\mathrm{dc}} Q$ control adopts DC voltage control; the outer loop of the PQ control adopts active and reactive power control; and the outer loop of the $U_{\text {ac }} f$ control adopts voltage and frequency control. The PI regulator is used in the closed-loop control.

The energy storage system adopts the double closedloop structure, the outer loop is power control, and the inner loop is current control. The PI regulator is used in the closed-loop control, and the control parameters are shown in Table 2 .

\section{Optimizing Operation Model of the Flexible Interconnected Distribution Network}

According to the comprehensive control strategy presented, the optimal operation model of the flexible interconnected distribution network based on SES-VSC-HVDC includes the distribution network optimization and the ESS SOC recovery.

4.1. Distribution Network Optimization. This paper develops the objective function with the lowest value of the comprehensive power supply cost from the perspective of the power sale enterprises. The comprehensive power supply cost consists of the purchase cost and the loss cost of distribution network. The purchase cost is related to the market spot price and the high-voltage transmission cost, which is reflected by the nodal price of the substation bus connected to the feeder [3]. Therefore, for the interconnected distribution network, the nodal price of different feeders is usually different.

The objective function of the comprehensive power supply cost is given below:

$$
\begin{aligned}
F_{1} & =\min \sum_{t=1}^{T}\left[f_{1}(t)+f_{2}(t)\right] \Delta t, \\
f_{1}(t) & =\sum_{i=1}^{n} C_{i}(t) P_{\mathrm{ST} i}(t), \\
f_{2}(t) & =C_{\mathrm{w}}(t)\left(\sum_{i=1}^{n} P_{\mathrm{ST} i}(t)+\sum_{i=1}^{n} P_{\mathrm{DG} i}(t)-\sum_{i=1}^{n} P_{\mathrm{D} i}(t)+P_{\mathrm{ESS}}(t)\right),
\end{aligned}
$$

where $n$ is the number of network nodes; $f_{1}(t)$ and $f_{2}(t)$ are, respectively, the purchase cost and the loss cost; $C_{i}(t), P_{\mathrm{ST} i}(t), P_{\mathrm{DG} i}(t)$, and $P_{\mathrm{D} i}(t)$ are, respectively, the bus nodal price, the power output of the substation, the active power output of distributed generation, and the active power of the load at the node $i$; and $C_{w}(t)$ is the electricity price of 
TABLE 2: PI regulator parameters in different control modes.

\begin{tabular}{lcc}
\hline Control mode & Outer loop control parameters & Inner loop control parameters \\
\hline \multirow{2}{*}{$U_{\mathrm{dc}} Q$ control } & $K_{p}=5$ & $K_{p}=100$ \\
\hline \multirow{2}{*}{ PQ control } & $K_{i}=300$ & $K_{i}=4000$ \\
\hline \multirow{2}{*}{$U_{\text {ac }} f$ control } & $K_{p}=0.005$ & $K_{p}=100$ \\
\hline \multirow{2}{*}{ ESS } & $K_{i}=0.625$ & $K_{i}=4000$ \\
& $K_{p}=3$ & $K_{p}=100$ \\
& $K_{i}=100$ & $K_{i}=4000$ \\
\hline
\end{tabular}

the network loss cost, which is generally equal to purchasing price $C_{i}(t)$ in numerical value.

On the basis of distribution network operation constraints, it is necessary to consider the active and reactive power of the substation (i.e., $P_{\mathrm{ST} i}(t)$ and $\left.Q_{\mathrm{ST} i}(t)\right)$, the active and reactive power output of the distributed generation (i.e.,
$P_{\mathrm{DG} i}(t)$ and $\left.Q_{\mathrm{DG} i}(t)\right)$, and the active and reactive power output of the SES-VSC-MTDC (i.e., $P_{k}^{i}(t)$ and $Q_{k}^{i}(t)$ ). The constraints of the optimization include the power flow equations, the power constraints of the substation, the voltage constraints, and the line capacity constraints, which are described by the following equation:

$$
\left\{\begin{array}{l}
P_{\mathrm{ST} i}(t)-P_{\mathrm{D} i}(t)+P_{\mathrm{DG} i}(t)-P_{k}^{i}(t)=U_{i}(t) \sum_{j=1}^{n-1} U_{j}(t)\left(G_{i j} \cos \delta_{i j}(t)+B_{i j} \sin \delta_{i j}(t)\right) \\
Q_{\mathrm{ST} i}(t)-Q_{\mathrm{D} i}(t)+Q_{\mathrm{DG} i}(t)-Q_{k}^{i}(t)=U_{i}(t) \sum_{j=1}^{n-1} U_{j}(t)\left(G_{i j} \sin \delta_{i j}(t)+B_{i j} \cos \delta_{i j}(t)\right) \\
0 \leq P_{\mathrm{ST} i}(t) \leq \bar{P}_{\mathrm{ST} i}, \\
Q_{\mathrm{ST} i} \leq Q_{\mathrm{ST} i}(t) \leq \bar{Q}_{\mathrm{ST} i} \\
\underline{U}_{i} \leq U_{i}(t) \leq \bar{U}_{i} \\
-\bar{S}_{i j} \leq S_{i j}(t) \leq \bar{S}_{i j}
\end{array}\right.
$$

where $U_{i}(t)$ and $U_{j}(t)$ are the voltage amplitudes of nodes $i$ and $j ; G_{i j}$ and $B_{i j}$ are, respectively, the mutual conductance and mutual susceptance between node $i$ and node $j ; \delta_{i j}(t)$ is the voltage phase difference between node $i$ and node; $Q_{D i}(t)$ is the reactive power of the load at node $i ; S_{i j}(t)$ is the line power transferred between node $i$ and node $j$; and superscript “" and subscript “_" represent the upper and lower limits of the variable.

The operation constraints of the SES-VSC-MTDC are given by (4)-(10).

4.2. ESS SOC Recovery. Control strategy III, aiming at minimizing absolute value from the current value to the middle value of SOC, is used to ensure that the ESS can be recovered to a moderate state as soon as possible in order to cope with the regulation problem in the future period. The objective function is given by

$$
F_{2}=\min \left|\operatorname{SOC}(t)-\frac{P_{\mathrm{ESS}} \Delta t}{S_{\mathrm{ESS}}}-\mathrm{SOC}_{\mathrm{mid}}\right|,
$$

where $\mathrm{SOC}_{\text {mid }}$ generally takes a value of around 0.5 [19]. Operation constraints for the SOC state recovery of the ESS are given by (7)-(10).

Considering the requirements of the recovery time and the standby state of the ESS, when SOC $(t)$ returns to the range of (14), the operation mode of the SOC state recovery is terminated.

$$
0.9 \mathrm{SOC}_{\mathrm{mid}} \leq \mathrm{SOC}(t) \leq 1.1 \mathrm{SOC}_{\mathrm{mid}}
$$

4.3. Model Solution. According to the SES-VSC-MTDC comprehensive control strategy proposed in this paper, there are three optimization models to be solved, i.e., the power regulation optimization, the power-energy timing regulation optimization, and the SOC state recovery. The power-energy timing regulation optimization and the SOC state recovery are used to determine the ESS active power output range with the SOC value. Then, the equality constraint of the active power balance becomes the inequality constraint, and thus the optimization models are in fact the nonlinear optimization problems. All of optimization problems are solved with the primal-dual interior-point method $[20,21]$.

\section{Case Studies and Simulation Results}

5.1. Case Scenarios. The proposed control strategy for the SES-VSC-MTDC is tested with a 33-bus system in Figure 3. The case system is a flexible interconnected distribution network including feeders from four different substations connected by the SES-VSC-MTDC. The rated voltage of the system is $10 \mathrm{kV}$. The line uses the YJV22-3*400 type cable used mainly by the urban distribution network in China [3]. The system topology parameters are shown in Table 3. Five 


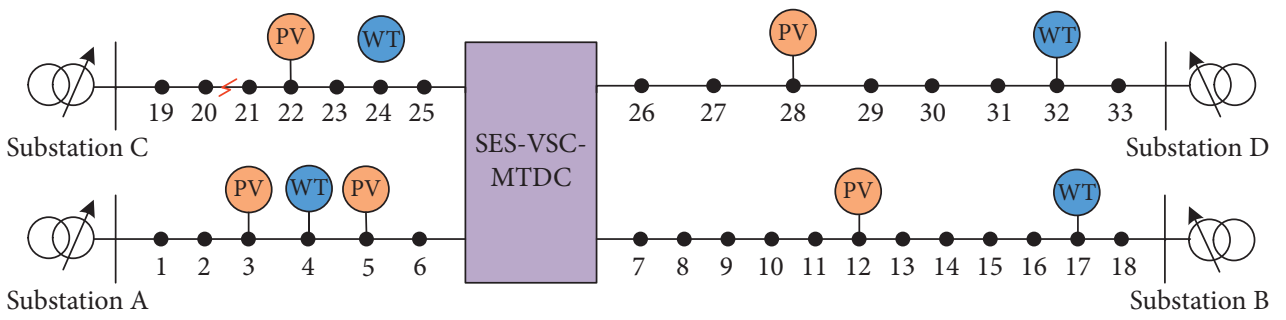

Figure 3: 33-bus system.

TABle 3: Parameters of the system.

\begin{tabular}{lcccc}
\hline Branch & Length $(\mathrm{km})$ & $R$ (p.u.) & $X$ (p.u.) & $B$ (p.u.) \\
\hline $1-2$ & 1.370333 & 0.023947 & 0.035034 & 0.005438 \\
$2-3$ & 1.116 & 0.019502 & 0.028532 & 0.004429 \\
$3-4$ & 1.971 & 0.034443 & 0.05039 & 0.007822 \\
$4-5$ & 0.964 & 0.016846 & 0.024646 & 0.003826 \\
$5-6$ & 1.392333 & 0.024331 & 0.035596 & 0.005526 \\
$7-8$ & 1.756 & 0.030686 & 0.044894 & 0.006969 \\
$8-9$ & 1.0915 & 0.019074 & 0.027905 & 0.004332 \\
$9-10$ & 1.4465 & 0.025278 & 0.036981 & 0.005741 \\
$10-11$ & 2.231 & 0.038987 & 0.057037 & 0.008854 \\
$11-12$ & 1.981 & 0.034618 & 0.050646 & 0.007862 \\
$12-13$ & 2.5635 & 0.044797 & 0.065538 & 0.010174 \\
$13-14$ & 1.877 & 0.032801 & 0.047987 & 0.007449 \\
$14-15$ & 2.0515 & 0.03585 & 0.052448 & 0.008142 \\
$15-16$ & 3.0915 & 0.054024 & 0.079037 & 0.012269 \\
$16-17$ & 2.7585 & 0.048205 & 0.070523 & 0.010948 \\
$17-18$ & 2.9875 & 0.052207 & 0.076378 & 0.011856 \\
$19-20$ & 1.6 & 0.02796 & 0.040905 & 0.00635 \\
$20-21$ & 1.007 & 0.017597 & 0.025745 & 0.003996 \\
$21-22$ & 1.281 & 0.022385 & 0.03275 & 0.005084 \\
$22-23$ & 1.45 & 0.025339 & 0.037071 & 0.005755 \\
$23-24$ & 0.862667 & 0.015075 & 0.022055 & 0.003424 \\
$24-25$ & 0.379667 & 0.006635 & 0.009707 & 0.001507 \\
$26-27$ & 0.857 & 0.014976 & 0.02191 & 0.003401 \\
$27-28$ & 1.147667 & 0.020055 & 0.029341 & 0.004555 \\
$28-29$ & 0.682667 & 0.01193 & 0.017453 & 0.002709 \\
$29-30$ & 0.364333 & 0.006367 & 0.009315 & 0.001446 \\
$30-31$ & 0.683333 & 0.011941 & 0.01747 & 0.002712 \\
$31-32$ & 0.962333 & 0.016817 & 0.024603 & 0.003819 \\
$32-33$ & 0.455667 & 0.007963 & 0.01165 & 0.001808 \\
\hline & & & &
\end{tabular}

sets of PV and four sets of WT are connected to the system, whose configuration parameters are shown in Table 4 . The peak-price period (07:00 19:00) and the valley-price period (19:00 07:00) of each bus node are shown in Table 5.

The rated capacity and loss factor of the VSC are, respectively, 4.5 MVA and 0.02. The configured ESS is $0.5 \mathrm{MW} / 1 \mathrm{MWh}$, the initial SOC value is $50 \%$, the upper and lower SOC safety limits are, respectively, $100 \%$ and $10 \%$, and the charge/discharge efficiency is $90 \%$. The interval of the substation power output is $0 \sim 8 \mathrm{MW}$ (power reversal is not allowed), the line capacity is $8 \mathrm{MVA}$, and the allowable voltage range of each node is [0.93, 1.07].

The three scenarios are compared and analyzed: (I) the system open-loop operation, (II) the flexible interconnection operation based on power regulation, and (III) the flexible interconnection operation based on power-energy timing regulation. The operation cycle is set to be $24 \mathrm{~h}$ that is divided into 96 time intervals. It is assumed that DG power output
TABle 4: Parameters of DG.

\begin{tabular}{lcc}
\hline DG & Location (node) & Capacity (MW) \\
\hline & 3 & 3 \\
& 5 & 3 \\
PV & 12 & 3 \\
& 22 & 3 \\
& 28 & 3 \\
& 4 & 6 \\
WT & 17 & 2 \\
& 24 & 1.5 \\
& 32 & 4 \\
\hline
\end{tabular}

TABle 5: Parameters of electricity price.

\begin{tabular}{lcc}
\hline Node & Valley price $(¥ / \mathrm{MWh})$ & Peak price $(¥ / \mathrm{MWh})$ \\
\hline Bus A & 310 & 610 \\
Bus B & 330 & 620 \\
Bus C & 368 & 658 \\
Bus D & 340 & 630 \\
\hline
\end{tabular}

and load power remain unchanged within $15 \mathrm{~min}$. The DG power output and load power of each feeder are shown in Figures 4-7. It is assumed that a fault occurs between nodes 20 and 21 on feeder $C$ at 14:30, the switches on both sides are disconnected, and the line fault is cleared at 16:00.

\subsection{Comparative Analysis of Operation Results}

Scenario I. During the open-loop operation, the DG power output of each feeder is greater than the load demand in multiple periods, and the distributed power cannot be fully accommodated, and wind and solar energy need to be abandoned by $12.25 \mathrm{MWh}$. During several time periods, the voltages of multiple nodes exceed the limit due to the large output of renewable energy, and wind and solar energy need to be abandoned by 3.23MWh, as shown in Tables 6 and 7 . During the fault period from 14:30 to 16:00, all nonfaulty areas of node 21's downstream on the feeder C are out of power supply. In addition, due to the natural distribution of power flow, the network loss and power purchase cost cannot be optimized, and thus the comprehensive power supply cost is high (Table 7). In summary, the open-loop operation cannot fully accommodate the renewable energy, and the power supply reliability and the operation economy are poor.

Scenario II. Under the flexible interconnection operation based on power regulation, the power flow of the distribution network becomes optimal, and the comprehensive power supply cost is 


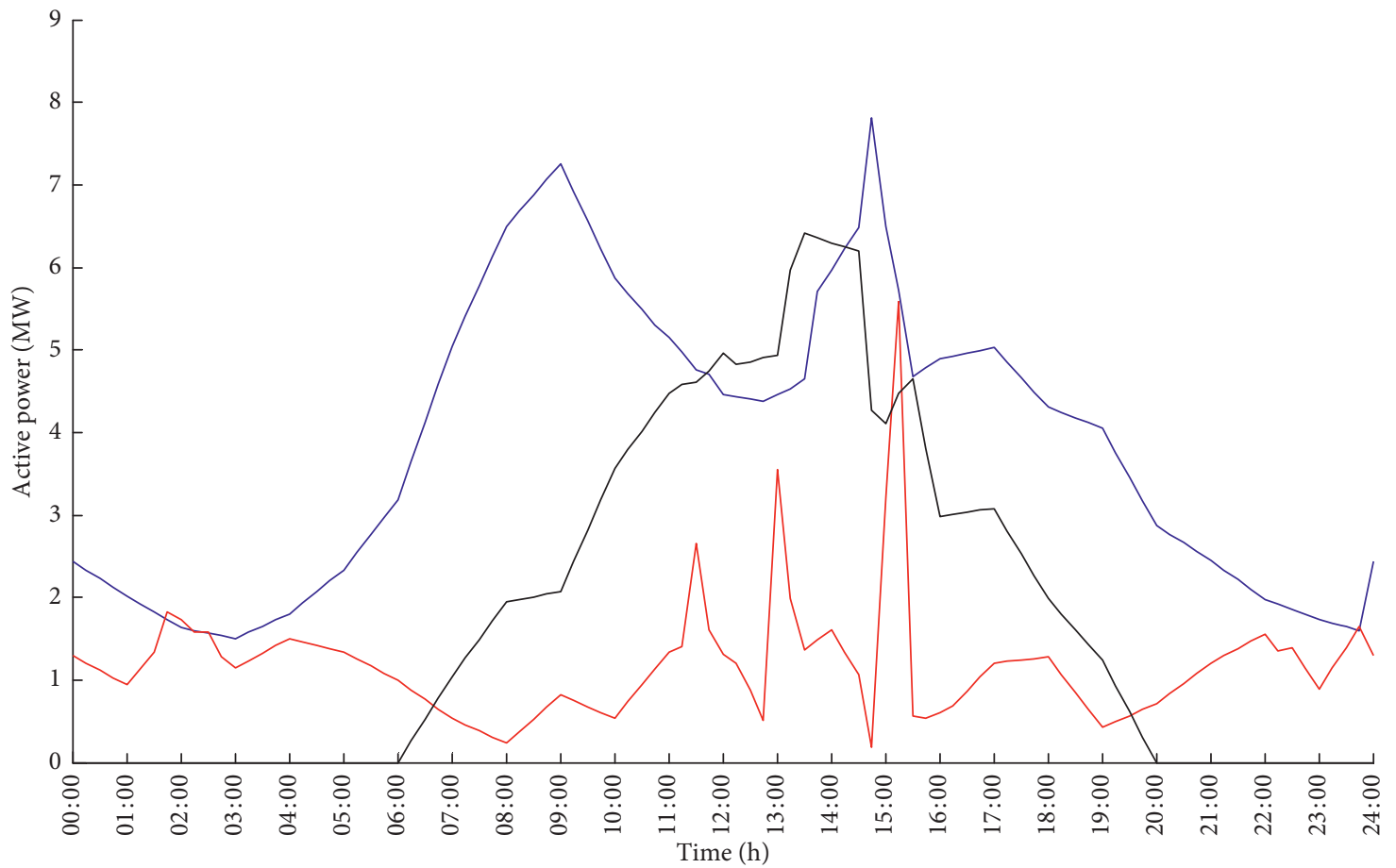

— Load of feeder A

Wind power of feeder $\mathrm{A}$

— Photovoltaic of feeder A

FIgure 4: Load and DG curves of feeder A.

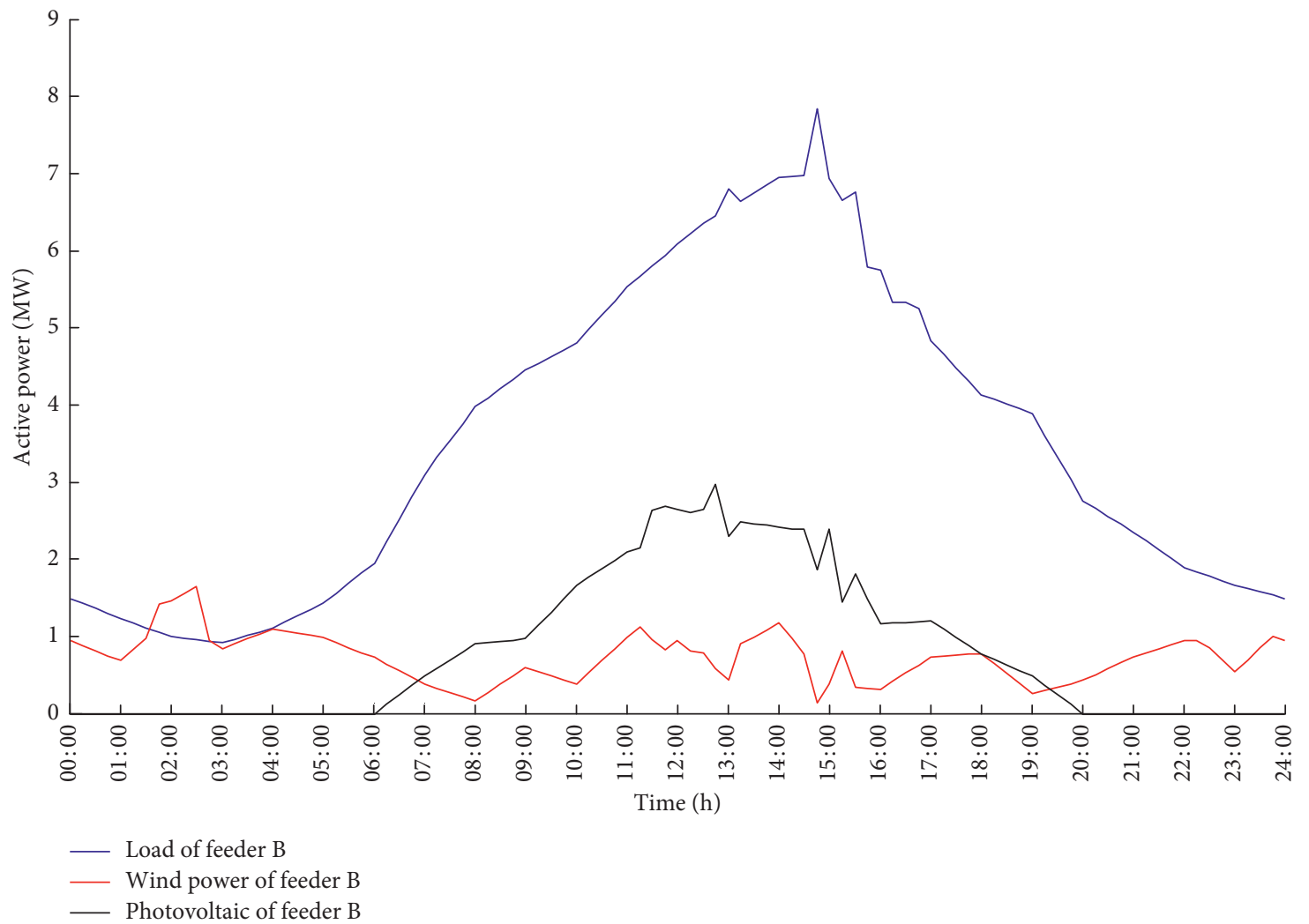

Figure 5: Load and DG curves of feeder B. 


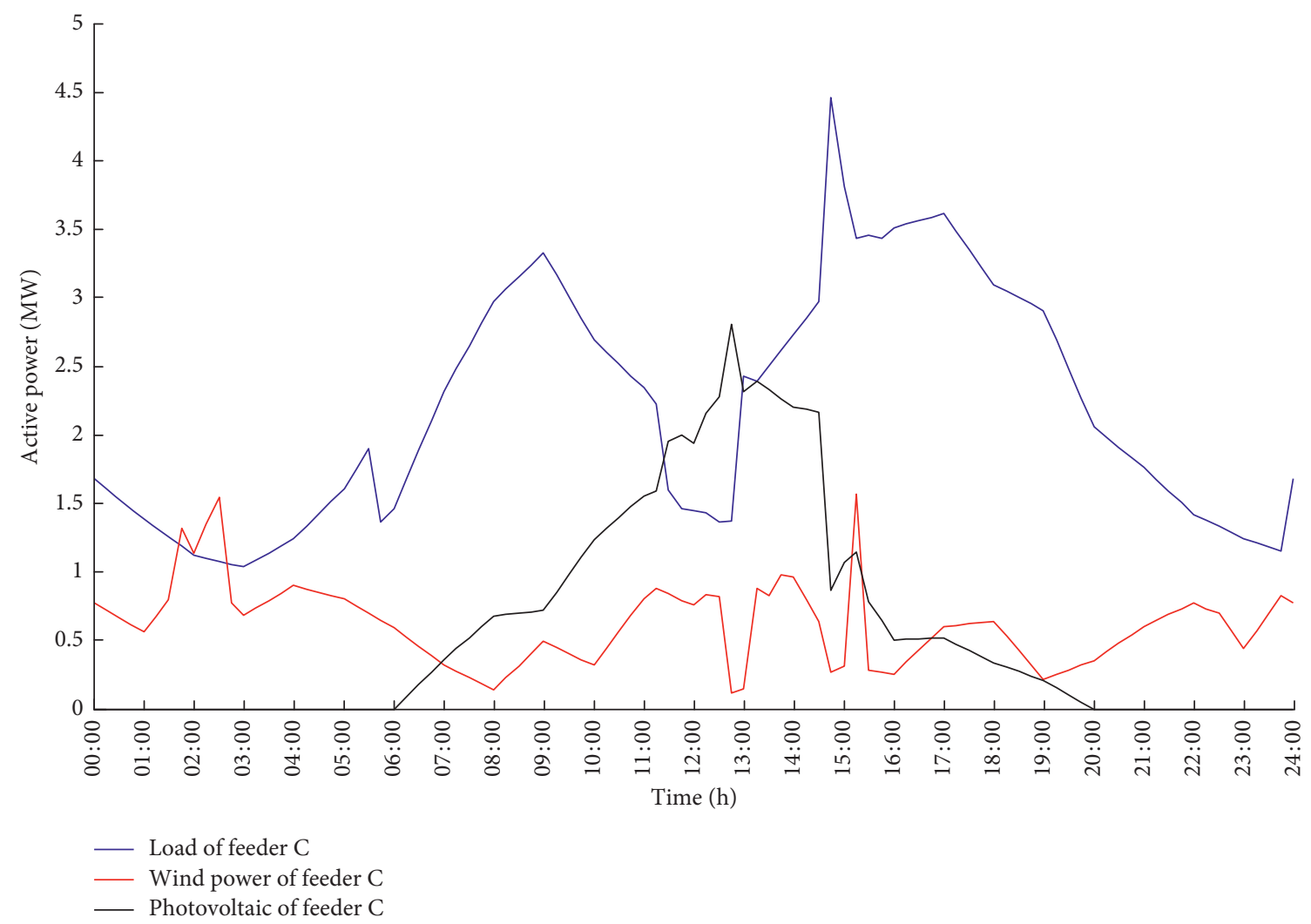

FIgure 6: Load and DG curves of feeder C.

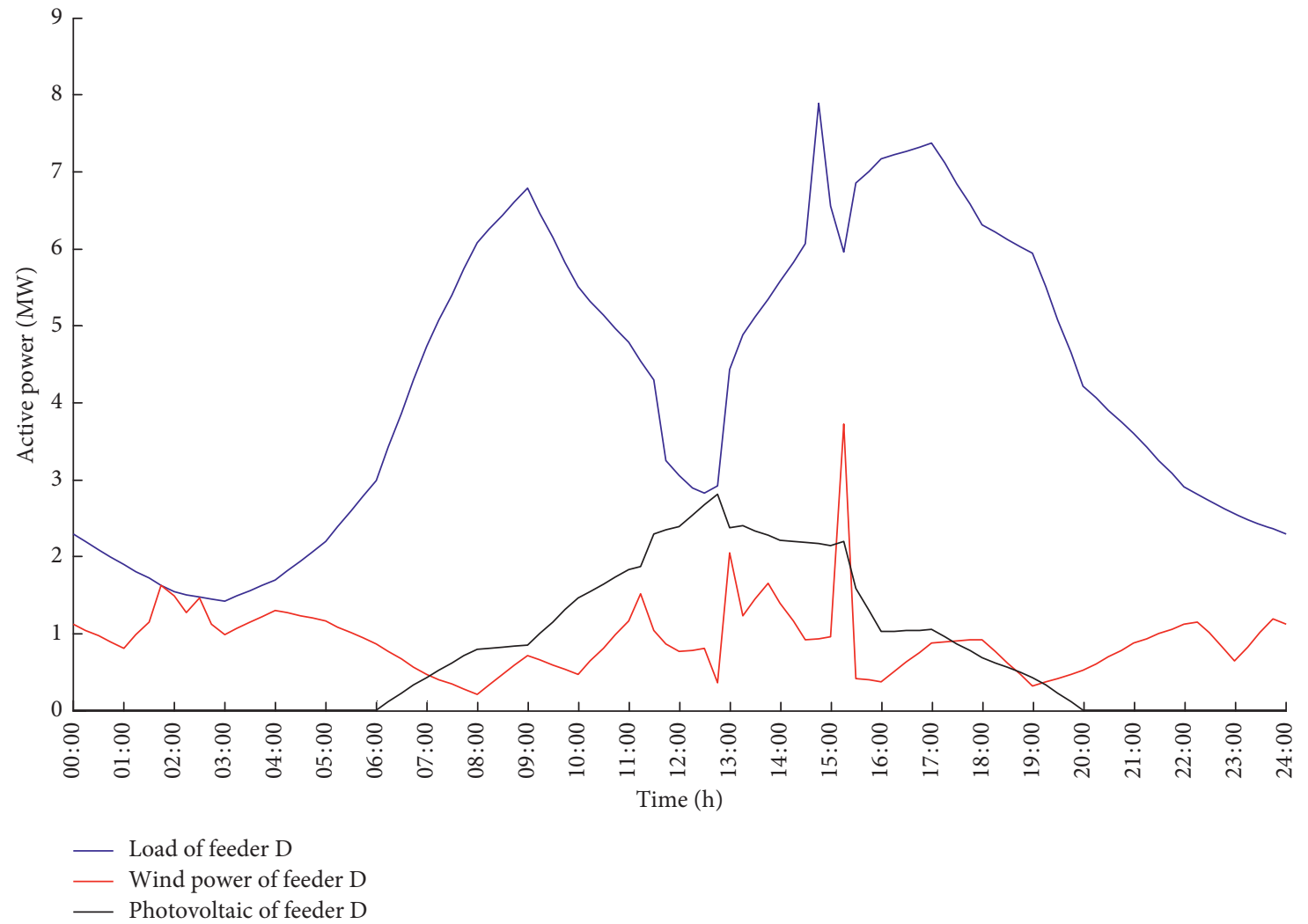

FIgURE 7: Load and DG curves of feeder D. 
TABLE 6: Analysis of no feasible solution of scenarios I and II.

\begin{tabular}{|c|c|c|}
\hline Scene & No-solution periods & Analysis \\
\hline \multirow{3}{*}{ I } & $01: 45 \sim 02: 45$ and $10: 45 \sim 15: 15$ & $\begin{array}{c}\text { DGs' outputs are greater than } \\
\text { loads (feeder) }\end{array}$ \\
\hline & $\begin{array}{l}\text { A: 10:45 14:30 and 15:00 15:30, B: 01:45 02:45, 13:00 13:15, and 14:30 15:30, C: } 02: 15 \sim 02: 30 \text {, } \\
\text { 11:30 12:45, and 13:15 14:00, and D: 12:00 12:45 }\end{array}$ & Voltage violation \\
\hline & $01: 45 \sim 02: 45$ and $11: 30 \sim 12: 30$ & $\begin{array}{c}\text { DGs' outputs are greater than } \\
\text { loads (system) }\end{array}$ \\
\hline II & $13: 00 \sim 13: 15,15: 15 \sim 15: 30$, and $14: 45 \sim 15: 00$ & $\begin{array}{l}\text { Voltage violation line power } \\
\text { violation }\end{array}$ \\
\hline
\end{tabular}

TABLE 7: Comparison of operation results of three scenarios.

\begin{tabular}{lccc}
\hline Scene & Reliability & Abandoned wind and solar (MWh) & Comprehensive power supply cost (¥) \\
\hline I & Poor & 15.48 & 102312.53 \\
II & Good & 0.27 & 86811.52 \\
III & Excellent & $\mathbf{0}$ & $\mathbf{8 5 5 7 9 . 4 6}$ \\
\hline
\end{tabular}

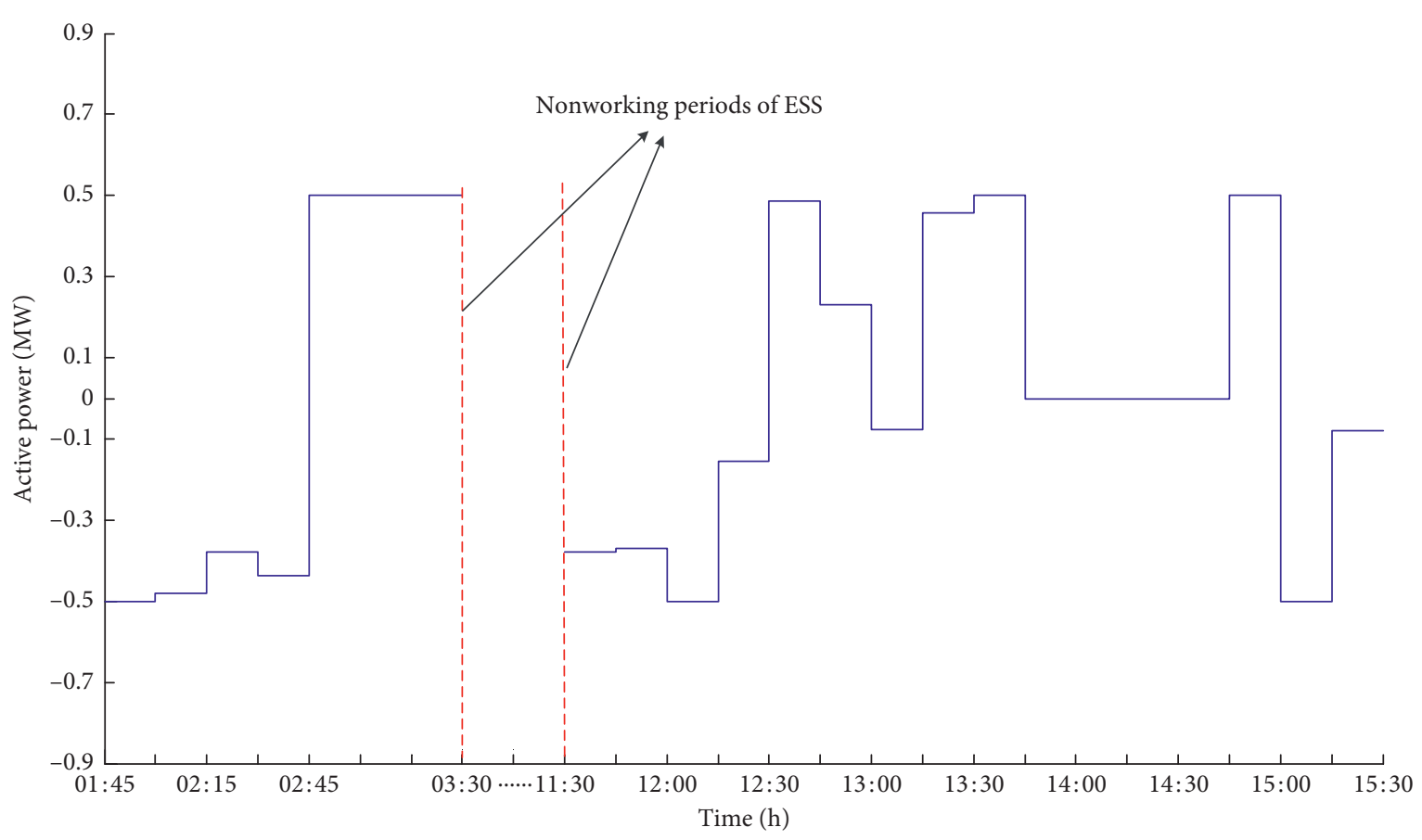

Figure 8: Charge-discharge power curves of ESS.

significantly reduced during the operation cycle (Table 7). The problems caused by the high penetration of renewable energy in multiple periods, such as the power reversal and voltage violation, can be effectively solved by the power regulation of interconnected feeders. However, during the time periods of 01 : 45-02:45, 11:30-12:30, and 13:00-13:15, there is still no solution due to constraint restrictions, as shown in Table 6. According to the analysis, during the time periods of 01:45-02:45 and 11: $30-12: 30$, the total DG power output in the system is greater than the total load, and the power cannot be reversed. During the time period of 13:00-13:15, the DG power output on feeder A is greater than the load, and the DG power output on feeders $\mathrm{C}$ and $\mathrm{D}$ is basically equal to the load, and the feed-in power is small. Therefore, the DG that is not consumed on feeder A needs to be transferred to feeder $\mathrm{B}$, which makes the partial node voltages on feeder B exceed the limit. Therefore, in the above periods, it is still necessary to abandon the wind and PV.

During the fault period of 14:30-16:00, the SES-VSCMTDC supplies power to the passive network on feeder $C$ by changing control mode, and the power supply from node 21 to all the downstream power-off loads is guaranteed. However, during the time periods of 14:45-15:00 and 15:15-15:30, there is no solution due to constraint restrictions. According to the analysis, during the time period of 14:45-15:00, the loads of feeders $\mathrm{A}, \mathrm{B}$, and $\mathrm{D}$ are heavy, and the available regulation capacity of these feeders is small due to the limited line capacity. The available regulation capacity of the feeders cannot meet the demand of loads on feeder C. During the time period of 15: 15-15:30, there is no available regulation capacity since feeder $\mathrm{C}$ is the passive network. The DG power output of feeder D is 


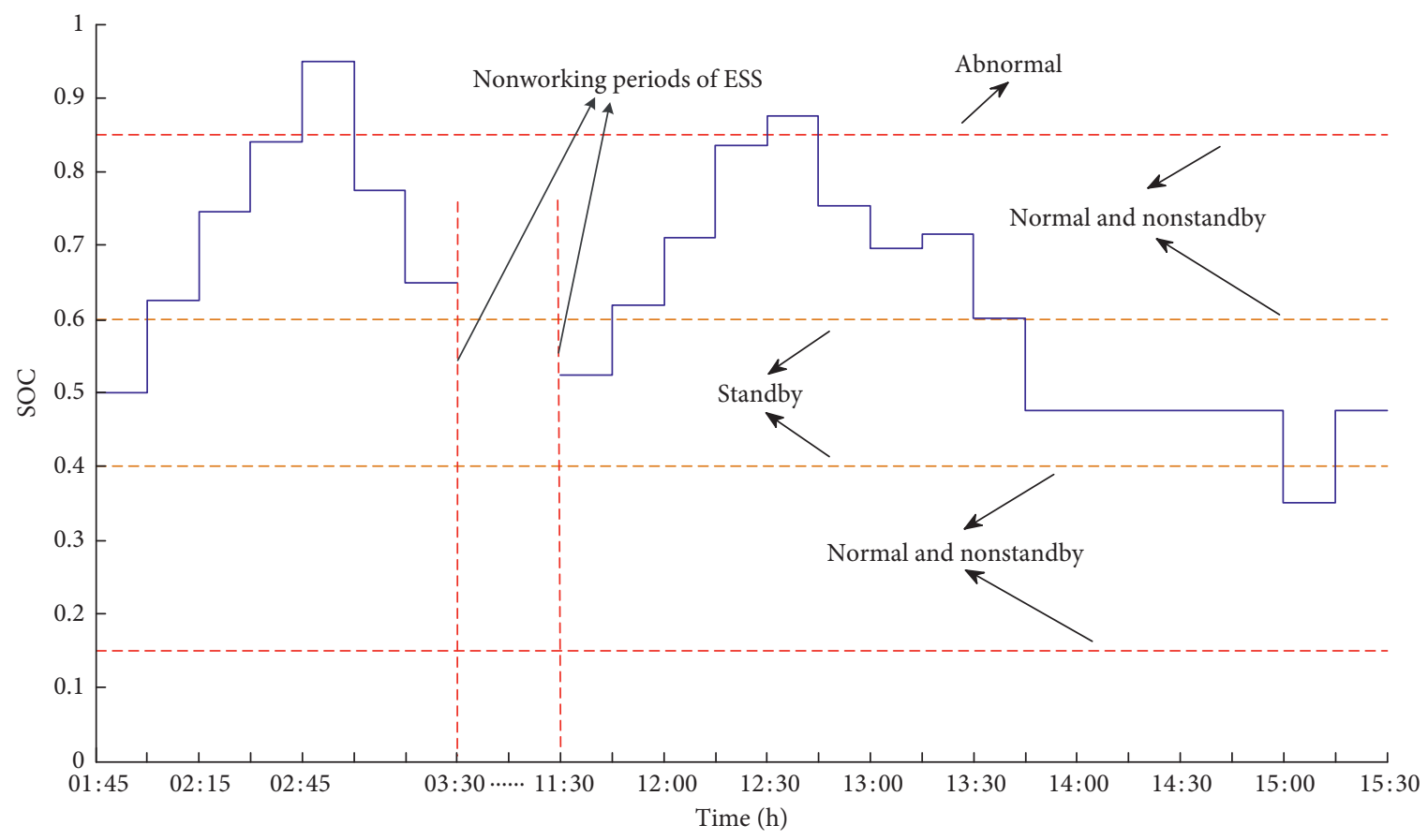

FIgURE 9: SOC variation curves of ESS.

basically equal to the load in numerical value. The additional DG power output on feeder A needs to be transferred to feeder $\mathrm{B}$, causing the partial node voltage to exceed the limit. Therefore, the abandonment of wind and solar energy is required during these two time periods.

In summary, in scenario II, when the feeder's available regulation capacity is sufficient, the power flow distribution can be effectively optimized to satisfy the limits from the power nonreversal constraints, the node voltage constraints, and the line capacity constraints. However, when the total available regulation capacity of each feeder of the system is insufficient during some specific time periods, the power regulation cannot meet the operation requirements, and energy storage is required for the power regulation.

Scenario III. The system has solutions during the aforementioned no-solution periods because the ESS participates in the optimization. Therefore, it is unnecessary to abandon wind and solar energy, and the comprehensive power supply cost is further reduced, as shown in Table 7.

The SES-VSC-MTDC can dynamically adjust power flows according to the change of the operation state of distribution network and can quickly respond to the power fluctuations from DGs and loads, which can make full use of renewable energy, improve power supply reliability, improve power supply quality, and reduce comprehensive power supply cost.

5.3. Control Strategy Analysis. The charge-discharge power curves and the SOC variation curves of the ESS are shown in Figures 8 and 9, respectively (only the working periods of the
ESS are selected). During the period of 01:45-02:45, the pretreatment calculation has no solution, and the ESS is put into operation to provide energy timing optimal regulation and absorb excess renewable energy. At time instant 02:45, the SOC is in the abnormal state. In order to cope with the case of next pretreatment's no solution, during the period of 02:45-03:30, the ESS runs the SOC recovery strategy and returns to the standby state. From 11:30 to 12:30, the pretreatment calculation has no solution, and the ESS is put into operation to absorb the additional renewable energy. If there is no SOC recovery strategy, the ESS will have no available regulation capacity during the period of 11:30-12:30. From 12:30 to 13:00, the ESS implements the SOC recovery strategy. At time instant 13:00, the pretreatment calculation has no solution again, and the ESS is in a normal and nonstandby state. To maintain the system operation, the ESS stops the SOC recovery and provides energy timing optimal regulation. During the period of 13:15-13:45, the ESS continues to operate the SOC recovery strategy and returns to a standby state. During the period of 14:45-15:00, the ESS injects power into the system, which compensates the power shortage of feeder C. At time instant 15:00, the SOC is in the normal and nonstandby state and is restored. The same operation strategy is applied during the time periods of 15 : 15-15:30 and 13:00-13:15.

It can be seen from the above analysis that the SES-VSCMTDC comprehensive control strategy can make use of the ESS in a reasonable SOC in each period of an operation cycle and participate in the necessary energy timing optimal regulation. The calculation shows that if the comprehensive control strategy is not adopted, the installed capacity of the ESS should be increased to $2.3 \mathrm{MWh}$. Meanwhile, the charging and discharging cycle number should be increased to 6 in order to achieve the optimal operation. 


\section{Conclusions}

A novel SES-VSC-MTDC modeling and optimization method is proposed for the interconnected distribution network in this paper. The contributions of this work are summarized as follows:

(1) The proposed SES-VSC-MTDC can effectively improve the penetration of the renewable energy, the power supply reliability, the power supply quality, and the operation economy, compared with the traditional open-loop operation distribution network and the interconnected distribution network with only power regulation based on VSC-MTDC.

(2) The proposed novel comprehensive control strategy for the SES-VSC-MTDC and the resulting optimal operation strategy of the flexible interconnected distribution network, which can effectively reduce the installed capacity of the energy storage, improve the operation lifespan of the energy storage and achieve the expected application value.

Furthermore, several valuable issues are worth being studied:

(1) Flexible loads (such as electric vehicles and intelligent air conditioners) and distributed energy storage should be considered in the optimization model. The scientific pricing mechanism and optimal control of flexible loads and distributed energy storage will help to reduce the capacity of SES-VSC-MTDC and improve the economy of the distribution network.

(2) The power output of other renewable energy reflects the characteristic of randomness, and the sudden power change inevitably brings trouble to the distribution network security. How to take uncertainty into account in the optimal operation model will be studied in the future.

\section{Abbreviations}

VSC: $\quad$ Voltage source converter

HVDC: $\quad$ High-voltage direct current

VSC-MTDC: Multiterminal back-to-back VSC-HVDC

SES-VSC- $\quad$ Self-energy storage-based multiterminal

MTDC: back-to-back VSC-HVDC

ESS: $\quad$ Energy storage system

SVG: $\quad$ Static VAR generator

SOP: $\quad$ Soft open point

SOC: $\quad$ State of charge.

\section{Appendix}

The OPF model is realized with the following nonlinear optimization model:

$$
\begin{cases}\min & f(\mathbf{x}) \\ \text { subject to } & \mathbf{h}(\mathbf{x})=0 \\ & \underline{g} \leq \mathbf{g}(\mathbf{x}) \leq \bar{g} .\end{cases}
$$

With the relaxation variables, the inequality constraints are transformed into equality constraints, and the Lagrange and obstacle functions are achieved. The functions are shown as follows:

$$
\begin{aligned}
L= & \mathbf{f}(\mathbf{x})-\mathbf{y}^{T} \mathbf{h}(\mathbf{x})-\mathbf{z}^{T}[\mathbf{g}(\mathbf{x})-\mathbf{l}-\underline{\mathbf{g}}] \\
& -\mathbf{w}^{T}[\mathbf{g}(\mathbf{x})+\mathbf{u}-\overline{\mathbf{g}}]-\mu \sum_{j=1}^{r} \ln \left(l_{j}\right)-\mu \sum_{j=1}^{r} \ln \left(u_{j}\right),
\end{aligned}
$$

where $\mathbf{y}$ is the Lagrange multiplier of the equality constraint; $\mathbf{z}$ and $\mathbf{w}$ are the Lagrange multipliers of the inequality constraint; $\mathbf{l}$ and $\mathbf{u}$ are the relaxation variables of the inequality constraint; and $\mu$ is the penalty factor.

The KTT condition of the problem can be obtained by solving the partial derivative of each variable:

$$
\left\{\begin{array}{l}
L_{x}=\nabla_{x} \mathbf{f}(\mathbf{x})-\nabla_{x} \mathbf{h}(\mathbf{x}) \mathbf{y}-\nabla_{x} \mathbf{g}(\mathbf{x})(\mathbf{z}+\mathbf{w})=0 \\
L_{y}=\mathbf{h}(\mathbf{x})=0 \\
L_{z}=\mathbf{g}(\mathbf{x})-\mathbf{l}-\underline{\mathbf{g}}=0 \\
L_{w}=\mathbf{g}(\mathbf{x})+\mathbf{u}-\overline{\mathbf{g}}=0 \\
L_{l}=\mathbf{z}-\mu \mathbf{L}^{-1} \mathbf{e}=0 \\
L_{u}=-\mathbf{w}-\mu \mathbf{U}^{-1} \mathbf{e}=0
\end{array}\right.
$$

The above equation is a set of nonlinear equations that can be solved by the Newton method. Based on the last two equations in equation (A.3), it is clear that

$$
\mu=\frac{\mathbf{l}^{T} \mathbf{z}-\mathbf{u}^{T} \mathbf{w}}{2 r} .
$$

By defining Gap $=\mathbf{l}^{T} \mathbf{z}-\mathbf{u}^{T} \mathbf{w}$, the following derivations are achieved:

$$
\mu=\sigma \frac{\mathrm{Gap}}{2 r},
$$

where $\sigma \in(0,1)$ is the central parameter that is usually equal to 0.1 , and the good convergence effect can be obtained in most cases. The equation is linearized to obtain the following iterative correction equation:

$$
\left[\begin{array}{cccccc}
\mathbf{H} & \nabla_{x} \mathbf{h}(\mathbf{x}) & \nabla_{x} \mathbf{g}(\mathbf{x}) & \nabla_{x} \mathbf{g}(\mathbf{x}) & 0 & 0 \\
\nabla_{x} \mathbf{h}(\mathbf{x})^{T} & 0 & 0 & 0 & 0 & 0 \\
\nabla_{x} \mathbf{g}(\mathbf{x})^{T} & 0 & 0 & 0 & -\mathbf{I} & 0 \\
\nabla_{x} \mathbf{g}(\mathbf{x})^{T} & 0 & 0 & 0 & 0 & \mathbf{I} \\
0 & 0 & \mathbf{L} & 0 & \mathbf{Z} & 0 \\
0 & 0 & 0 & \mathbf{U} & 0 & \mathbf{W}
\end{array}\right]\left[\begin{array}{c}
\Delta \mathbf{x} \\
\Delta \mathbf{y} \\
\Delta \mathbf{z} \\
\Delta \mathbf{w} \\
\Delta \mathbf{l} \\
\Delta \mathbf{u}
\end{array}\right]=\left[\begin{array}{c}
\mathbf{L}_{x} \\
-\mathbf{L}_{y} \\
-\mathbf{L}_{z} \\
-\mathbf{L}_{w} \\
-\mathbf{L}_{l}^{\mu} \\
-\mathbf{L}_{u}^{\mu}
\end{array}\right],
$$

where $\mathbf{H}=-\left[\nabla_{x}^{2} \mathbf{f}(\mathbf{x})-\nabla_{x}^{2} \mathbf{h}(\mathbf{x}) \mathbf{y}-\nabla_{x}^{2} \mathbf{g}(\mathbf{x})(\mathbf{z}+\mathbf{w})\right]$. As the dimension of the coefficient matrix of the modified equation is large, it is difficult to be solved. Therefore, three small matrices are obtained with the matrix decomposition, and then the iteration is carried out. The derivations are shown as follows: 


$$
\begin{aligned}
{\left[\begin{array}{cc}
\mathbf{H}^{\prime} & \nabla_{x} \mathbf{h}(\mathbf{x}) \\
\nabla_{x}^{T} \mathbf{h}(\mathbf{x}) & 0
\end{array}\right]\left[\begin{array}{c}
\Delta \mathbf{x} \\
\Delta \mathbf{y}
\end{array}\right] } & =\left[\begin{array}{c}
\mathbf{L}_{x}^{\prime} \\
-\mathbf{L}_{y}
\end{array}\right], \\
{\left[\begin{array}{cc}
\mathbf{I} & \mathbf{L}^{-1} \mathbf{Z} \\
0 & \mathbf{I}
\end{array}\right]\left[\begin{array}{c}
\Delta \mathbf{z} \\
\Delta \mathbf{l}
\end{array}\right] } & =\left[\begin{array}{c}
-\mathbf{L}^{-1} \mathbf{L}_{l}^{\mu} \\
\mathbf{L}_{\mathbf{z}}+\nabla_{x}^{T} \mathbf{g}(\mathbf{x}) \Delta \mathbf{x}
\end{array}\right], \\
{\left[\begin{array}{cc}
\mathbf{I} & \mathbf{U}^{-1} \mathbf{W} \\
0 & \mathbf{I}
\end{array}\right]\left[\begin{array}{c}
\Delta \mathbf{w} \\
\Delta \mathbf{u}
\end{array}\right] } & =\left[\begin{array}{c}
-\mathbf{U}^{-1} \mathbf{L}_{\mathbf{u}}^{\mu} \\
-\mathbf{L}_{\mathbf{w}}-\nabla_{x}^{T} \mathbf{g}(\mathbf{x}) \Delta \mathbf{x}
\end{array}\right] .
\end{aligned}
$$

Then,

$$
\begin{aligned}
\mathbf{L}^{-1} \mathbf{Z} & =\operatorname{diag}\left(\frac{z_{1}}{l_{1}}, \ldots, \frac{z_{i}}{l_{i}}\right), \\
\mathbf{U}^{-1} \mathbf{W} & =\operatorname{diag}\left(\frac{w_{1}}{l_{1}}, \ldots, \frac{w_{i}}{l_{i}}\right) .
\end{aligned}
$$

The coefficient matrix of iterative correction equation is mainly composed of the equality constrained Jacobian matrix $\nabla_{x} \mathbf{h}(\mathbf{x})$, the inequality constrained Jacobian matrix $\nabla_{x} \mathbf{g}(\mathbf{x})$, the diagonal matrix $\mathbf{L}^{-1} \mathbf{Z}$ and $\mathbf{U}^{-1} \mathbf{W}$, and the Hessian matrix $\mathbf{H}^{\prime}$. The control variables of SES-VSC-
MTDC in control strategies II and III increase the energy storage power output based on control strategy I, and the corresponding charging and discharging power constraint and SOC constraint are added to the constraint conditions. The following is the formation of the coefficient matrix of the modified equation in control strategies II and III. Control strategy I only needs to delete the relevant variables and constraints on this basis, and relevant content is not repeated here.

State variable:

$$
\widetilde{\mathbf{x}}=\left\{\begin{array}{ll}
\theta_{i} & U_{i}
\end{array}\right\}
$$

Control variables:

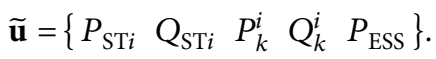

Total system variables:

$$
\mathbf{x}=\left\{\begin{array}{lllllll}
P_{\mathrm{ST} i} & Q_{\mathrm{ST} i} & P_{k}^{i} & Q_{k}^{i} & P_{\mathrm{ESS}} & \theta_{i} & U_{i}
\end{array}\right\} .
$$

According to the equality constraints in equations (4), (8), and (12), each node is constrained by two power flow equations:

$$
\left.\begin{array}{l}
\Delta P_{\mathrm{i}}=\sum_{k \in i} P_{\mathrm{ST} k}+\sum_{k \in i} P_{\mathrm{DG} k}-P_{\mathrm{D} i}-P_{k}^{i}-U_{i} \sum_{j=1}^{n-1} U_{j}\left(G_{i j} \cos \delta_{i j}+B_{i j} \sin \delta_{i j}\right)=0 \\
\Delta Q_{\mathrm{i}}=\sum_{k \in i} Q_{\mathrm{ST} k}+\sum_{k \in i} Q_{\mathrm{DG} k}-Q_{\mathrm{D} i}-\mathrm{Q}_{k}^{i}-U_{i} \sum_{j=1}^{n-1} U_{j}\left(G_{i j} \sin \delta_{i j}-B_{i j} \cos \delta_{i j}\right)=0
\end{array}\right\}
$$

where $k \in i$ means the $k$-th generator is connected to node I.

The constraints of SES-VSC-MTDC output power equation are shown as follows:

$$
\Delta P_{k}=\sum_{k=1}^{N_{\text {VSC }}} P_{k}+\sum_{k=1}^{N_{\text {VSC }}}\left(A_{k} P_{k}\right)+P_{\text {ESS }}=0 .
$$

Thus, the following equality constrained Jacobian matrix can be obtained:

$$
\nabla_{x} \mathbf{h}(\mathbf{x})=\left[\begin{array}{c}
\frac{\partial \mathbf{h}}{\partial \mathbf{P}_{\mathrm{ST}}} \\
\frac{\partial \mathbf{h}}{\partial \mathbf{P}_{\mathbf{k}}} \\
\frac{\partial \mathbf{h}}{\partial \mathbf{Q}_{\mathrm{ST}}} \\
\frac{\partial \mathbf{h}}{\partial \mathbf{Q}_{\mathbf{k}}} \\
\frac{\partial \mathbf{h}}{\partial \widetilde{\mathbf{x}}}
\end{array}\right]=\left[\begin{array}{lll}
\frac{\partial \Delta \mathbf{P}}{\partial \mathbf{P}_{\mathrm{ST}}} & \frac{\partial \Delta \mathbf{Q}}{\partial \mathbf{P}_{\mathrm{ST}}} & \frac{\partial \Delta \mathbf{P}_{\mathbf{k}}}{\partial \mathbf{P}_{\mathrm{ST}}} \\
\frac{\partial \Delta \mathbf{P}}{\partial \mathbf{P}_{\mathbf{k}}} & \frac{\partial \Delta \mathbf{Q}}{\partial \mathbf{P}_{\mathbf{k}}} & \frac{\partial \Delta \mathbf{P}_{\mathbf{k}}}{\partial \mathbf{P}_{\mathbf{k}}} \\
\frac{\partial \Delta \mathbf{P}}{\partial \mathbf{Q}_{\mathrm{ST}}} & \frac{\partial \Delta \mathbf{Q}}{\partial \mathbf{Q}_{\mathrm{ST}}} & \frac{\partial \Delta \mathbf{P}_{\mathbf{k}}}{\partial \mathbf{Q}_{\mathrm{ST}}} \\
\frac{\partial \Delta \mathbf{P}}{\partial \mathbf{Q}_{\mathbf{k}}} & \frac{\partial \Delta \mathbf{Q}}{\partial \mathbf{Q}_{\mathbf{k}}} & \frac{\partial \Delta \mathbf{P}_{\mathbf{k}}}{\partial \mathbf{Q}_{\mathbf{k}}} \\
\frac{\partial \Delta \mathbf{P}}{\partial \widetilde{\mathbf{x}}} & \frac{\partial \Delta \mathbf{Q}}{\partial \widetilde{\mathbf{x}}} & \frac{\partial \Delta \mathbf{P}_{\mathbf{k}}}{\partial \widetilde{\mathbf{x}}}
\end{array}\right] .
$$

According to the inequality constraints shown in equations (4), (8), and (12), the following inequality constrained Jacobian matrices can be obtained:

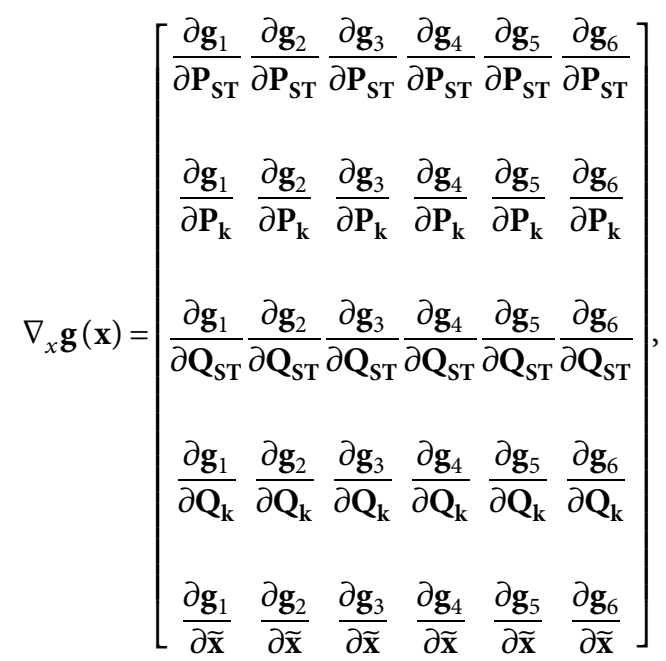

where $\mathbf{g}_{1}$ is the upper and lower limits of active power output of substation, distributed generation, and SES-VSC-MTDC; $\mathbf{g}_{2}$ is the upper and lower limits of reactive power output of substation, distributed generation, and SES-VSC-MTDC; $\mathbf{g}_{3}$ is the upper and lower limits of node voltage; $\mathbf{g}_{4}$ is the upper and lower limits of line capacity; $\mathbf{g}_{5}$ is the output capacity 
limit of converter; and $\mathbf{g}_{6}$ is the charging and discharging power limit of ESS.

Hessian array:

$$
\begin{aligned}
\mathbf{H}^{\prime}= & -\nabla_{x}^{2} \mathbf{f}(\mathbf{x})+\nabla_{x}^{2} \mathbf{h}(\mathbf{x}) \mathbf{y}+\nabla_{x}^{2} \mathbf{g}(\mathbf{x})(\mathbf{z}+\mathbf{w}) \\
& -\nabla_{x} \mathbf{g}(\mathbf{x})\left[\mathbf{L}^{-1} \mathbf{Z}-\mathbf{U}^{-1} \mathbf{W}\right] \nabla_{x}^{T} \mathbf{g}(\mathbf{x}) .
\end{aligned}
$$

\section{Data Availability}

The data used to support the findings of this study are available from the corresponding author upon request.

\section{Conflicts of Interest}

The authors declare that there are no conflicts of interest regarding the publication of this paper.

\section{Acknowledgments}

This study was supported in part by the National Natural Science Foundation of China (51707089) and in part by the Science and Technology Project of State Grid Jiangsu Electric Power Co., Ltd. (J2018084).

\section{References}

[1] M. Fan, K. Sun, W. Gu, Z. Li, and F. Zhang, "A novel generation rescheduling algorithm to improve power system reliability with high renewable energy penetration," IEEE Transactions on Power Systems, vol. 33, no. 3, pp. 3349-3357, 2018.

[2] A. K. Singh and S. K. Parida, "A review on distributed generation allocation and planning in deregulated electricity market," Renewable and Sustainable Energy Reviews, vol. 82, no. 3, pp. 4132-4141, 2018.

[3] L. Ge, W. Lu, X. Yuan, Y. Hu, and B. Hu, "Back-to-back VSCHVDC based loop-closed optimal operation for active distribution network," Automation of Electric Power Systems, vol. 41, no. 6, pp. 135-141, 2017.

[4] C. Zhang, A. Zhang, Y. Cui, and T. Wen, "An advanced control and extensible configuration for static var generator," Mathematical Problems in Engineering, vol. 2016, Article ID 2095973, 9 pages, 2016.

[5] A. I. Nousdilis, G. C. Christoforidis, and G. K. Papagiannis, "Active power management in low voltage networks with high photovoltaics penetration based on prosumers' self-consumption," Applied Energy, vol. 229, pp. 614-624, 2018.

[6] Q. Qi, J. Wu, and C. Long, "Multi-objective operation optimization of an electrical distribution network with soft open point," Applied Energy, vol. 208, pp. 734-744, 2017.

[7] H. Ji, C. Wang, P. Li et al., "An enhanced SOCP-based method for feeder load balancing using the multi-terminal soft open point in active distribution networks," Applied Energy, vol. 208, pp. 986-995, 2017.

[8] P. Li, H. Ji, H. Yu et al., "Combined decentralized and local voltage control strategy of soft open points in active distribution networks," Applied Energy, vol. 241, pp. 613-624, 2019.

[9] M. B. Shafik, G. I. Rashed, and H. Chen, "Optimizing energy savings and operation of active distribution networks utilizing hybrid energy resources and soft open points: case study in sohag, Egypt," IEEE Access, vol. 8, pp. 28704-28717, 2020.
[10] D. Sciano, A. Raza, R. Salcedo, M. Diaz-Aguilo et al., "Evaluation of DC links on dense-load urban distribution networks," IEEE Transactions on Power Delivery, vol. 31, no. 3, pp. 1317-1326, 2016.

[11] W. Liu and Y. Xu, "Reliability modeling of MMC-based flexible interconnection controller considering the uncertainty of current loading," Microsystem Technologies, vol. 25, no. 3, pp. 905-916, 2018.

[12] L. Bai, T. Jiang, F. Li, H. Chen, and X. Li, "Distributed energy storage planning in soft open point based active distribution networks incorporating network reconfiguration and DG reactive power capability," Applied Energy, vol. 210, 13 pages, 2017.

[13] C. Wang, G. Song, P. Li et al., "Research and prospect for soft open point based flexible interconnection technology for smart distribution network," Automation of Electric Power Systems, vol. 40, no. 22, pp. 168-175, 2016.

[14] F. Attanasio, S. Wasterlein, T. Pidancier et al., "Low voltage soft open point with energy storage: system simulation and prototype preliminary test results," in Proceedings of the International Symposium on Power Electronics, Electrical Drives, Automation and Motion (SPEEAM), pp. 254-261, Amalf, Italy, June 2018.

[15] H. Ji, C. Wang, P. Li, J. Zhao, G. Song, and J. Wu, "Quantified flexibility evaluation of soft open points to improve distributed generator penetration in active distribution networks based on difference-of-convex programming," Applied Energy, vol. 218, pp. 338-348, 2018.

[16] C. Yao, C. Zhou, J. Yu, K. Xu, P. Li, and G. Song, "A sequential optimization method for soft open point integrated with energy storage in active distribution networks," Energy Procedia, vol. 145, pp. 528-533, 2018.

[17] L. Ge, W. Zhang, X. Yuan, J. Chen, and L. Zhong, "Control strategy of self-energy storage based multi-terminal back-toback VSC-HVDC system," Electric Power Automation Equipment, vol. 39, no. 12, pp. 14-20, 2019.

[18] R. Sandano, M. Farrell, and M. Basu, "Enhanced master/slave control strategy enabling grid support services and offshore wind power dispatch in a multi-terminal VSC HVDC transmission system," Renewable Energy, vol. 113, pp. 15801588, 2017.

[19] Q. Xu, J. Xiao, X. Hu, P. Wang, and M. Y. Lee, “A decentralized power management strategy for hybrid energy storage system with autonomous bus voltage restoration and state-ofcharge recovery," IEEE Transactions on Industrial Electronics, vol. 64, no. 9, pp. 7098-7108, 2017.

[20] S. J. Wright, Primal-Dual Interior-Point Methods, Society for Industrial and Applied Mathematics, Philadelphia, PA, USA, 1997.

[21] M. I. Khan and K. Xia, "Effective self adaptive multiple source localization technique by primal dual interior point method in binary sensor networks," IEEE Communications Letters, vol. 21, no. 5, pp. 1119-1122, 2017. 